\title{
Autorin
}

Stefanie Kurt, Assistenzprofessorin FH (Tenure Track), Dr. iur., Hochschule für Soziale Arbeit, HES-SO Valais-Wallis, Route de la Plaine 2, 3960 Siders, 00412760691 00, stefanie.kurt@hevs.ch ${ }^{1}$

\section{Der Zugang zu Bildung für geflüchtete Personen in der Schweiz}

Seitenzahl: 14

Deadline 28. November 2018

Erscheint in: RdJB Recht der Jugend und des Bildungswesens, https://www.rdjb.nomos.de/

\footnotetext{
${ }^{1}$ Diese Publikation wurde im Rahmen des NCCR on the move, finanziert durch den Schweizerischen Nationalfonds und der HES-SO Valais-Wallis verfasst. Die Autorin dankt herzlich Daniela Duff, Dozentin FH, Hochschule für Soziale Arbeit, HES-SO Valais-Wallis, Dr. Andrea Friedli, Wissenschaftliche Mitarbeiterin FH, Hochschule für Soziale Arbeit, HES-SO Valais-Wallis, Dr. Katrin Sontag, Universität Basel, und Lisa Probst für die wertvollen Anregungen und die kritische Durchsicht des vorliegenden Artikels.
} 


\section{Der Zugang zu Bildung für geflüchtete Personen in der Schweiz}

\section{Einleitung}

Geflüchtete Personen in der Schweiz treffen auf eine komplexe Situation, wenn es um den Zugang $\mathrm{zu}$ Bildung geht. Dies hat mit dem Zusammenspiel zwischen den drei unterschiedlichen Rechtsbereichen, Bildung, Asyl und Integration, sowie der föderalen Ausgestaltung der Kompetenzen, zu tun. Im folgenden Artikel wird die grundlegende rechtliche Situation dargestellt, sowie die speziellen Bereiche der frühen Förderung, respektive die Angebote für Kinder im Vorschulalter ( 0 bis 4-Jährige), der obligatorische Grundschulunterricht (4 bis 15-Jährige), die nachobligatorische (Aus)Bildung (ab 16 Jahren) und die Berufsbildung (ab 16 Jahren) thematisiert. Schliesslich fasst der Artikel die wichtigsten Aspekte zusammen.

\section{Ein föderales Geflecht: Bildung, Asyl und Integration}

Die schweizerische Bildungslandschaft ist geprägt durch die Mehrsprachigkeit und den Föderalismus. Grundsätzlich bestimmt Art. 61a Art. 1 BV, dass Bund und Kantone gemeinsam im Rahmen ihrer Zuständigkeiten für eine hohe Qualität und Durchlässigkeit des Bildungsraumes Schweiz sorgen. Dennoch tragen die 26 Kantone grundsätzlich die Hauptverantwortung im Bildungsbereich $^{2}$, mit Ausnahme der nachobligatorischen Schule, wo sowohl die Kantone als auch der Bund Zuständigkeiten haben. ${ }^{3}$ Hinsichtlich der Berufsbildung und der Weiterbildung erlässt der Bund Vorschriften. ${ }^{4}$ Das Bildungssystem Schweiz ist grundsätzlich durchlässig und so sind verschiedene Aus- und Weiterbildungswege sowohl im schulischen wie beruflichen Bereich möglich. ${ }^{5}$ Diese Kompetenzverteilung im Bildungsbereich ist nun mit den ebenfalls föderalen Eigenheiten des schweizerischen Asylverfahrens respektive mit dem Ausländerinnen-, Ausländerund Integrationsgesetz ${ }^{6}$ konfrontiert. $^{7}$

Eine ausländische Person, welche in der Schweiz ein Asylgesuch stellt, wird zuerst in einem Empfangs- und Verfahrenszentrum auf Bundesebene, geleitet durch das Staatssekretariat für Migration, untergebracht. ${ }^{8}$ Asylsuchende unterliegen während den ersten drei Monaten einem Arbeitsverbot. ${ }^{9}$ Jedoch kann der Bund für Personen, welche sich in diesen Zentren aufhalten, Beiträge

\footnotetext{
${ }^{2}$ Vgl. Art. 62 Abs. 1 Bundesverfassung der Schweizerischen Eidgenossenschaft vom 18. April 1999 (BV), SR 101.

${ }^{3}$ Vgl. Art. 63a Abs. 2 und Abs. 3 BV und Webseite der Schweizerischen Konferenz der kantonalen Erziehungsdirektoren, www.edk.ch, Bildungslandschaft Schweiz (07.02.2019).

${ }^{4}$ Vgl. Art. 63 BV und Art. 64a BV.

${ }^{5}$ Vgl. die Visualisierung der schweizerischen Bildungslandschaft:

https://www.sbfi.admin.ch/sbfi/de/home/bildung/bildungsraum-schweiz/das-duale-system.html (07.02.2019).

${ }^{6}$ Bundesgesetz über die Ausländerinnen und Ausländer und über die Integration, (Ausländer- und Integrationsgesetz, AIG) vom 16. Dezember 2005, SR 142.20.

${ }^{7}$ Literatur zum schweizerischen Asylverfahren respektive Migrationsrecht, u.a.: Caroni, M., Scheiber, N., Preisig, C., Zoeteweij, M., Migrationsrecht, 4. Auflage, Stämpfli AG, Bern, 2018, Schweizerische, Flüchtlingshilfe SFH, Handbuch zum Asyl- und Wegweisungsverfahren, 2., vollständig überarbeitete Auflage, Haupt Verlag, Bern, 2015, Literatur zur Bildungsforschung: Engelage, S., (Hrsg.), Migration und Berufsbildung in der Schweiz, Seismo Verlag, Zürich, 2018. ${ }^{8}$ Aktuell wird das Asylverfahren in der Schweiz neustrukturiert und beschleunigt. Die Umsetzung erfolgt ab März 2019. Weitere Informationen sind hier zu finden: https://www.sem.admin.ch/sem/de/home/asyl/beschleunigung.html (07.02.2019).

${ }^{9}$ Vgl. Art. 43 Abs. 1 AsylG.
} 
für die Durchführung von Beschäftigungsprogrammen ausrichten. ${ }^{10}$ Diese Beschäftigungsprogramme sollen die positive Wahrnehmung von Asylsuchenden durch die lokale Bevölkerung fördern und gleichzeitig Ansammlungen von Asylsuchenden im öffentlichen Raum vorbeugen. Die Möglichkeit der Teilnahme an gemeinnützigen Tätigkeiten ermöglicht eine Tagesstruktur und wird durch finanzielle oder materielle Leistungen entschädigt. Diese Beschäftigungsprogramme dürfen aber in keiner Weise mit der Privatwirtschaft konkurrenzieren. ${ }^{11}$ Nach maximal 90 Tagen in einem Empfangs- und Verfahrenszentrum werden die Asylsuchenden in eine kantonale Unterkunft transferiert. ${ }^{12}$ Nun ist der Kanton respektive die Gemeinde für die Unterbringung, die Begleitung und Betreuung der Asylsuchenden zuständig. Dies beinhaltet auch den Zugang zu Bildung, insbesondere den Besuch der öffentlichen Schule für schulpflichtige Kinder ${ }^{13}$. Zudem lancierte der Bundesrat am 18. Dezember 2015 Begleitmassnahmen zur Umsetzung der am 9. Februar 2014 angenommenen Volksinitiative «gegen Masseneinwanderung» ${ }^{14}$, welche die Finanzierung einer intensiven und frühen Sprachförderung für 800-1000 Asylsuchende mit Bleibeperspektive pro Jahr seit dem 1. Januar 2018 beinhaltet. ${ }^{15}$ Art. 121 a BV verlangt unter anderem, dass die Schweiz die Zuwanderung selbständig steuert, sowie die Zahl der Bewilligungen für den Aufenthalt von Ausländerinnen und Ausländern durch jährliche Höchstzahlen und Kontingente begrenzt. Im Zuge der Umsetzung sprach sich der Bundesrat, wie auch das Parlament, dafür aus, insbesondere das inländische Arbeitskräftepotential auszuschöpfen, um die Zahl der Zuwanderinnen und Zuwanderer in die Schweiz zu begrenzen. ${ }^{16}$ Dies führte bereits zu einem frühen Zeitpunkt der Umsetzungsdebatten zur Idee, dass Asylsuchende mit hoher Bleibewahrscheinlichkeit frühzeitig eine der Ortsprachen erlernen sollen. ${ }^{17}$

Während der Dauer des Asylverfahrens haben Asylsuchende den Ausweis N, welcher das Recht auf Anwesenheit in der Schweiz bescheinigt. Es handelt sich jedoch nicht um eine ausländerrechtliche Bewilligung, sondern vielmehr bestimmt der Verfahrensstand des Asylverfahrens die Aufenthaltsberechtigung. Nach einem rechtskräftigen abgelehnten Asyl- und Wegweisungsentscheid muss die betroffene Person die Schweiz verlassen, unabhängig davon, ob der Ausweis N noch ein

\footnotetext{
${ }^{10}$ Vgl. Art. 91 Abs.4bis Asylgesetz vom 26. Juni 1998 (AsylG), SR 142.31. Der Bund schliesst hier

Leistungsvereinbarungen mit dem Standortkanton, der Standortgemeinde oder dem beauftragten Dritten ab. Für Personen, welche eine Arbeitsbewilligung haben oder an einem Beschäftigungsprogramm teilnehmen, unterliegen dem Arbeitsverbot nicht (Art. 43 Abs. 4 AsylG).

${ }^{11}$ Vgl. Staatssekretariat für Migration, Handbuch Asyl und Rückkehr, Artikel C1 Die Empfangs- und Verfahrenszentren, Pkt. 2.2.4, https://www.sem.admin.ch/dam/data/sem/asyl/verfahren/hb/c/hb-c1-d.pdf (07.02.2019).

12 Spescha, M., Kerland, A., Bolzli, P., Handbuch zum Migrationsrecht, 3., unveränderte Auflage, Orell Füssli Verlag, Zürich, 2015, S. 401.

${ }^{13}$ Vgl. Art. 62 Abs. 2 BV.

${ }^{14} \mathrm{Vgl}$. Art. 121a BV.

${ }^{15}$ Staatssekretariat für Migration, Rundschreiben, Eingabe Pilotprogramm «Frühzeitige Sprachförderung», BernWabern, 27. März 2017, https:/www.sem.admin.ch/dam/data/sem/integration/ausschreibungen/2018integrvorlehre/20170327-rs-fsf-d.pdf (07.02.2019).

${ }^{16}$ Vgl. Graf, A.-L., Mahon, P., Article 121a de la Constitution et accès au marché du travail, in: Jusletter 13. August 2018.

${ }^{17}$ Vgl. Bundesrat, Begleitmassnahmen Artikel 121a BV: Verstärkung der Integrationsmassnahmen für Flüchtlinge und vorläufig Aufgenommene, Bericht des Bundesrates in Erfüllung des Postulats Tornare (14.3523) vom 19. Juni 2014: Integration von Migrantinnen und Migranten in den schweizerischen Arbeitsmarkt, Bern, 18. Dezember 2015, S. $27 \mathrm{ff}$.
} 
gültiges Datum trägt. ${ }^{18}$ Asylsuchende, welche einen positiven Asylentscheid erhalten, werden als Flüchtlinge anerkannt und erhalten von ihrem Aufenthaltskanton den Aufenthaltstatus B (Aufenthaltsbewilligung). ${ }^{19}$ Die Erteilung einer Niederlassungsbewilligung richtet sich nach dem ausländerrechtlichen Verfahren. ${ }^{20}$ Asylsuchende, welche die Flüchtlingseigenschaft nicht erfüllen oder vom Asyl ausgeschlossen sind, werden aus der Schweiz weggewiesen. Jedoch muss hier das Staatssekretariat für Migration prüfen, ob dem Vollzug der Wegweisung nicht völkerrechtliche oder humanitäre Schranken entgegenstehen. Sofern dies der Fall ist, tritt die vorläufige Aufnahme (Ausweis F) als Ersatzmassnahme, daher keine Gewährung des Flüchtlingsstatus, an die Stelle des Wegweisungsvollzugs. Personen mit vorläufiger Aufnahme entsprechen nicht genau demjenigen Personenkreis, welche im Europäischen Recht unter den subsidiären Schutz ${ }^{21}$ fallen. Die vorläufige Aufnahme greift in einigen Fällen, wie beispielsweise bei der Unmöglichkeit des Wegweisungsvollzugs, bei einer medizinischen Notlage oder bei schwerwiegenden sozialen Probleme im Herkunftsland, während der subsidiäre Schutz gemäss EU-Recht nicht zur Anwendung kommt. Schliesslich ist mit dem subsidiären Schutz eine bessere Rechtsstellung im Aufnahmestaat verknüpft, als mit der vorläufigen Aufnahme. Während mit der Anerkennung des subsidiären Schutzes die sofortige Aufnahme einer Erwerbstätigkeit zu gestatten ist ${ }^{22}$, müssen Arbeitgeber den zuständigen Behörden die Arbeitsaufnahme einer vorläufigen Person bei der zuständigen Behörde melden $^{23}$. Sofern eine Person mit vorläufiger Aufnahme den Unterhalt nichts selbständig bestreiten kann, erhält diese Person Sozialhilfeleistungen. Der Ansatz für die Unterstützung liegt unter demjenigen der einheimischen Bevölkerung. Nach Möglichkeit hat diese Unterstützung in Form von Sachleistungen zu erfolgen. ${ }^{24}$ Schliesslich wird die Ersatzmassnahme vorläufige Aufnahme für höchstens ein Jahr erteilt und jährlich erneuert, sofern keine Beendigungsgründe vorliegen. ${ }^{25}$ Ein Anspruch auf die Erteilung einer Aufenthaltsbewilligung besteht nicht. ${ }^{26}$ Schliesslich können vorläufige Aufgenommene nach drei Jahren einen Antrag auf Familiennachzug stellen, wobei hier verschiedene Voraussetzungen, wie gemeinsames Wohnen, das Vorhandensein einer bedarfsgerechten Wohnung, Verständigung der am Wohnort gesprochenen Sprache und kein Bezug von Sozialhilfe und Ergänzungsleistungen, zu erfüllen sind. Ein Rechtsanspruch besteht jedoch nicht $^{27}$

Zusätzlich gibt es noch den Status der vorläufigen Aufnahme mit Flüchtlingsstatus. Darunter fallen Personen, welche den Flüchtlingsstatus nach völkerrechtlichen Vorgaben erfüllen, deren Asylgesuch

\footnotetext{
${ }^{18}$ Vgl. Spescha, M., Kerland, A., Bolzli, P., (Anm. 11), S. 400.

19 Art. 60 Abs. 1 AsylG.

${ }^{20}$ Art. 60 Abs. 2 AsylG, Art. 34 AIG.

${ }^{21}$ Vgl. Richtlinie 2011/95/EU des Europäischen Parlaments und des Rates vom 13. Dezember 2011 über Normen für die Anerkennung von Drittstaatsangehörigen oder Staatenlosen als Personen mit Anspruch auf internationalen Schutz, für einen einheitlichen Status für Flüchtlinge oder für Personen mit Anrecht auf subsidiären Schutz und für den Inhalt des zu gewährenden Schutzes.

${ }^{22}$ Vgl. Art. 26 Richtlinie 2011/95/EU.

${ }^{23}$ Vgl. 85a Abs. 2 AIG.

${ }^{24}$ Vgl. Art. 86 Abs. 1 AIG.

${ }^{25}$ Vgl. Art. 84 und Art. 84 AIG.

${ }^{26}$ Vgl. Art. 84 Abs. 5 AIG.

${ }^{27}$ Vgl. 85 Abs. 7 AIG. Vgl. dazu auch den Bericht des Bundesrates in Erfüllung der Postulate 11.3954, 13.3844, 14.3008, Vorläufige Aufnahme und Schutzbedürftigkeit: Analyse und Handlungsoptionen, 2016, ab S. 37 , https://www.sem.admin.ch/dam/data/sem/aktuell/news/2016/2016-10-14/ber-va-d.pdf (07.02.2019).
} 
jedoch abgelehnt wurde. ${ }^{28}$ Die Nichtgewährung der Flüchtlingseigenschaft erfolgt gestützt auf drei Asylausschlussgründen, die der Schweizer Gesetzgeber festgelegt hat. Es sind dies die Asylunwürdigkeit ${ }^{29}$, subjektive Nachfluchtgründe ${ }^{30}$ sowie Ausnahmesituationen bei erhöhten Spannungen, bewaffneten Konflikten oder ausserordentlich grossem Zustrom von Asylsuchenden ${ }^{31}$. Diese Personen verbleiben «vorläufig» in der Schweiz bis die Vollzugshindernisse wegfallen. ${ }^{32}$ Diese Personengruppe erhält daher teilweise Rechte gemäss der Genfer Flüchtlingskonvention. So erhalten vorläufig aufgenommene Flüchtlinge die gleichen Sozialhilfeansätze wie die einheimische Bevölkerung $^{33}$, haben jedoch keinen Anspruch auf Familiennachzug ${ }^{34}$ und unterliegen der Meldepflicht für die Aufnahme einer Erwerbstätigkeit ${ }^{35}$.

Sofern das Asylgesuch abgelehnt und eine Wegweisungsverfügung erlassen wurde, muss die betroffene Person die Schweiz verlassen. Definitiv abgewiesene Asylsuchende haben somit kein Anwesenheitsrecht mehr in der Schweiz. Dies führt unter anderem zum Ausschluss aus der Sozialhilfe, so dass diese Personen lediglich Nothilfe, Sachleistungen oder kleine finanzielle Beiträge und/oder Gutscheine erhalten. ${ }^{36}$ Diese Personen werden unter dem Begriff «Sans-Papiers» zusammengefasst. Darunter fallen beispielsweise Asylsuchende, deren Asylgesuch rechtskräftig abgewiesen wurde und die untergetaucht sind, um einer Ausschaffung zu entgehen oder auch abgewiesene Asylsuchende in den Nothilfestrukturen. Schliesslich können aber auch irregulär eingereiste Personen oder Personen mit ungültigen (abgelaufenen) Visa und/oder Aufenthaltstitel, die in der Schweiz bleiben, darunter gezählt werden. ${ }^{37}$

Als letzter Punkt in diesem föderalen Geflecht des Bildungszugangs und Asylverfahrens ist noch auf die Charakteristika der schweizerischen Integrationspolitik respektive derer rechtlichen Grundlagen kurz einzugehen. Integration wird als eine Querschnittsaufgabe verstanden, welche von allen staatlichen Organen auf allen föderalen Ebenen wahrzunehmen ist. ${ }^{38}$ Ziel der Integration ist die Schaffung von «günstigen Rahmenbedingungen für die Chancengleichheit und die Teilhabe der ausländischen Bevölkerung am öffentlichen Leben» ${ }^{39}$. In erster Linie hat Integration über die

\footnotetext{
${ }^{28}$ Vgl. Spescha, Kerland Bolzli, (Anm. 11), S. 378f.

${ }^{29}$ Vgl. Art. 53 AsylG.

${ }^{30}$ Vgl. Art. 54 AsylG.

${ }^{31}$ Vgl. Art. 55 AsylG.

${ }^{32}$ Vgl. Spescha, Kerland Bolzli, (Anm. 11), S. 378, 379.

${ }^{33}$ Vgl. Art. 86 Abs. 1 Satz 4 AIG.

${ }^{34}$ Vgl. Art. 85 Abs. 7 AIG.

${ }^{35}$ Vgl. Art. 85a Abs. 2 AIG.

${ }^{36}$ Vgl. Spescha, Kerland Bolzli, (Anm. 11), S. 403.

${ }^{37} \mathrm{Vgl}$. B, S, S. Volkswirtschaftliche Beratung in Kooperation mit SFM und Universität Genf, Sans-Papiers in der Schweiz 2015, Basel, 12. Dezember 2015, https:/www.sem.admin.ch/dam/data/sem/internationales/illegalemigration/sans_papiers/ber-sanspapiers-2015-d.pdf (07.02.2019), Efionayi-Mäder, D., Schönenberger, S., Steiner, I., Leben als Sans-Papiers in der Schweiz. Entwicklungen 2000-2010, Materialen zur Migrationspolitik, Eidgenössische Kommission für Migrationsfragen EKM, Bern, 2010, https:/www.ekm.admin.ch/dam/data/ekm/dokumentation/materialien/mat_sanspap_d.pdf (07.02.2019).

38 Art. 53 Abs. 4 AIG; Art. 2 Abs. 2 Verordnung vom 15. August 2018 über die Integration von Ausländerinnen und Ausländern (VIntA), SR 142.205.

39 Art. 53 Abs. 2 AIG.
} 
Regelstrukturen, unter anderem auch über die Schulen und die Berufsbildung, zu erfolgen. ${ }^{40}$ In zweiter Linie erfolgt die Integration über spezifische Massnahmen, welche die vorhandenen Lücken in den Regelstrukturen schliessen. Hierunter fallen die kantonalen Integrationsprogramme (KIP) und weitere Projekte. ${ }^{41}$ Schliesslich führt Art. 4 Abs. 2 AIG aus, dass die Integration längerfristig rechtmässig anwesenden Ausländerinnen und Ausländern die Teilhabe am wirtschaftlichen, sozialen und kulturellen Leben der Gesellschaft ermöglichen soll. ${ }^{42}$ In der Konsequenz sind also ausländische Personen ohne (rechtmässigen) Aufenthaltsstatus, wie (abgewiesene) Asylsuchende, nicht eine anvisierte Gruppe der schweizerischen Integrationspolitik. ${ }^{43}$ Allerdings hat der Erwerb von Bildung, insbesondere der obligatorische Besuch des Grundschulunterrichts durch Art. 62 Abs. 2 BV eine Sonderstellung. ${ }^{44}$

\section{Kinder im Vorschulalter}

Eine vorschulische Bildung und die frühe Förderung von Kindern ist weder durch die Bundesverfassung noch durch die kantonalen Gesetze rechtlich gewährleistet. Trotzdem lancierte das Staatssekretariat für Migration gemeinsam mit der Eidgenössischen Migrationskommission ein Modellvorhaben «Integrationsförderung im Frühbereich» bereits im Jahr 2009. Ziel war die Erreichbarkeit der Angebote für Eltern zu verbessern, die Anzahl der teilnehmenden ausländischen Kinder zu erhöhen und Betreuungspersonen interkulturell zu qualifizieren. ${ }^{45}$ Bis 2011 wurden so rund 86 innovative Projekte mit 5 Millionen Schweizer Franken unterstützt. ${ }^{46}$ Dennoch haben sich nur wenige Kantone im Bereich vorschulische Bildung respektive Frühförderung engagiert. ${ }^{47}$ Erst durch die Einführung der kantonalen Integrationsprogramme ${ }^{48}$ ab dem 1. Januar 2014 und der erhöhten Wahrnehmung der vorschulischen Bildung für geflüchtete Personen, haben die Kantone in den letzten Jahren gezielt Projekte initiiert und umgesetzt. Der im Jahr 2016 erschienene Zwischenbericht zu den kantonalen Integrationsprogrammen 2014-2017 hält im Bereich Frühförderung fest, dass viele Kantone auf eine qualitative gute frühe Sprachförderung setzen, jedoch das Erreichen von Familien

\footnotetext{
40 Art. 2 Abs. 3 VIntA.

${ }^{41} \mathrm{Vgl}$. https://www.sem.admin.ch/sem/de/home/themen/integration/foerderung.html (07.02.2019).

${ }^{42}$ Vgl. hier auch Art. 121a Abs. 3 BV, welcher die Integrationsfähigkeit als Kriterium für die Erteilung der Aufenthaltsbestimmung auflistet.

${ }^{43}$ Achermann, A., Amarelle, C., Art. 4 AuG, in: Amarelle und Nguyen (Hrsg.), Code annoté en droit des migrations, Volume II: Loi sur les étrangers, Stämpfli Verlag AG Bern, 2017, S. 24-44, Achermann, A.,

Art. 4 AuG, in: Caroni, Gächter, Turnherr (Hrsg.), Bundesgesetz über die Ausländerinnen Stämpflis Handkommentar, Stämpfli Verlag, Bern, 2010, N 25.

${ }^{44}$ Wintsch, S., Flüchtlingskinder und Bildung - Rechtliche Aspekte, ZStöR, Züricher Studien zum öffentlichen Recht Band/Nr. 182, Schulthess Juristische Medien AG, Zürich, 2008, S. 40.

${ }^{45}$ Vgl. Schulte-Haller M., Frühe Förderung, Forschung, Praxis und Politik im Bereich der Frühförderung: Bestandesaufnahme und Handlungsfelder, Materialien zur Migrationspolitik, Eidgenössische Kommission für Migrationsfragen EKM, Bern, 2009.

${ }^{46}$ Vgl. https://www.sem.admin.ch/sem/de/home/themen/integration/themen/fruehfoerderung.html (07.02.2019).

${ }^{47}$ Vgl. Bericht der Caritas Schweiz, Mit Chancengleichheit gegen Armut, Eine Analyse der Frühen Förderung in den Kantonen, Beobachtungen der Caritas zur Armutspolitik, 2013, https://www.caritas.ch/fileadmin/user_upload/Caritas_Schweiz/data/site/was-wir-sagen/unsereposition/positionspapiere/2014-und-aelter/Caritas_Armutsmonitoring_2013_DE.pdf (07.02.2019), Zur Situation in den Städten, siehe INFRAS im Auftrag der Jacobs Foundation, Situationsanalyse zur frühen Förderung in kleinen und mittleren Städten der Schweiz, Schlussbericht, Zürich, 31. Januar 2012, https://www.sem.admin.ch/dam/data/sem/integration/themen/fruehfoerd/ber-infras-fruehfoerderung-d.pdf (07.02.2019).

${ }^{48}$ Sog. KIP, Informationen sind zu finden auf: www.kip-pic.ch (07.02.2019).
} 
mit Förderbedarf sich als schwierig herausstellt. Ebenso haben die Kantone in den vergangenen Jahren die Mitarbeitenden gezielt auf interkulturelle Kompetenzen geschult ${ }^{49}$ und den Fokus auf den chancengerechten Zugang für Migrationsfamilien gelegt. Wöchentliche Hausbesuche zur präventiven Förderung wurden etabliert und die Zusammenarbeit mit der Mütter- und Väterberatung intensiviert. Hindernisse bilden aber nach wie vor die zielführende Information der Eltern und die Finanzierung der entsprechenden Angebote. ${ }^{50}$

Gemäss den aktuellen kantonalen Integrationsprogrammen (2017-2021) werden im Bereich der schulischen Frühförderung zwei Ziele verfolgt. Einerseits werden Migrationsfamilien gezielt über die Erwartungen im Bereich Kindererziehung im schweizerischen Kontext und über entsprechende Angebote (Gesundheit, Familienunterstützung und Integrationsförderung) informiert und gleichzeitig der Zugang zu diesen ermöglicht, andererseits arbeiten die verschiedenen Akteurinnen und Akteure engmaschig und koordiniert zusammen. ${ }^{51}$ Diese spezifischen Massnahmen zielen in erster Linie auf Kinder mit einem Flüchtlingsstatus respektive einer vorläufigen Aufnahme ab, im Unterschied zu Kindern, welche sich noch im laufenden Asylverfahren befinden. Die kürzlich lancierte Integrationsagenda Schweiz nennt jedoch das Wirkungsziel, dass beim Start der obligatorischen Schulzeit 80\% der Kinder aus dem Asylbereich, welche im Alter von 0-4 Jahren in die Schweiz gekommen sind, sich in der Sprache am Wohnort verständigen können sollen. Offen bleibt, ob die sprachliche Frühförderung bereits während dem Asylverfahren stattfindet oder erst nach dem positiven Asylentscheid respektive der vorläufigen Aufnahme startet. ${ }^{52}$ Jedoch gibt es diverse zivilgesellschaftliche Engagements, insbesondere in den Empfangs- und Verfahrenszentren auf Bundesebene, welche durch Treffpunkte oder der Organisation anderer Aktivitäten auch für Kinder im Vorschulalter Angebote schaffen. ${ }^{53}$

\section{Obligatorischer Grundschulunterricht}

Die Kantone sind für einen ausreichenden obligatorischen Grundschulunterricht zuständig. Die Schulpflicht dauert elf Jahre (Kindergarten, Primarstufe und Sekundarstufe) ${ }^{54}$ und steht allen Kindern offen $^{55}$. Art. 62 Abs. 2 BV präzisiert, dass der Grundschulunterricht unter staatlicher Leitung oder Aufsicht steht. Zudem ist der Anspruch auf ausreichenden und unentgeltlichen Grundschulunterricht

\footnotetext{
49 Staatssekretariat für Migration, Kantonale Integrationsprogramme 2014-2017, Zwischenbericht, Oktober 2016, S. 15, 28f., https://www.sem.admin.ch/dam/data/sem/integration/berichte/kip/2016/zwber-kip-2016-d.pdf (07.02.2019).

${ }^{50}$ Staatssekretariat für Migration, (Anm. 38), S. 28f.

${ }^{51}$ Vgl. www.kip-pic.ch/de/kip/fruehe-kindheit/ (07.02.2019).

${ }^{52}$ Vgl. Eidgenössisches Departement für Wirtschaft, Bildung und Forschung WBF, Konferenz der Kantonsregierungen, Schweizerische Konferenz der kantonalen Erziehungsdirektoren, Konferenz der kantonalen Sozialdirektorinnen und Sozialdirektoren, Integrationsagenda Schweiz, Bericht der Koordinationsgruppe vom 1. März 2018, S.11, https:/www.sem.admin.ch/dam/data/sem/integration/agenda/ber-koordinationsgruppe-integrationsagenda-d.pdf (07.02.2019).

53 Beispielsweise: https://www.agathu.ch/projekte/gestalten-mit-kindern/ (07.02.2019), eine Übersicht ist zu finden auf http://www.plattform-ziab.ch/engagement/portraits/ (07.02.2019).

${ }^{54}$ Vgl. Webseite der Schweizerischen Konferenz der kantonalen Erziehungsdirektoren, www.edk.ch, Bildungslandschaft Schweiz (07.02.2019). Die Primarstufe, inklusive zwei Jahre Kindergarten respektive Eingangsstufe umfasst acht Jahren und die Sekundarstufe I umfasst drei Jahre. Ausnahme bildet der Kanton Tessin, wo die Sekundarstufe I vier Jahre dauert. Die Kinder sind in der Regel vier Jahre alt.

${ }^{55}$ Vgl. Art. 62 Abs. 2 BV.
} 
gewährleistet. ${ }^{56}$ Dieser Art. $19 \mathrm{BV}$ ist somit eines der wenigen sozialen Grundrechte, welche einen einklagbaren verfassungsmässigen Anspruch auf eine staatliche Leistung begründen, wobei Grundrechtsträger das Kind ist. ${ }^{57}$ Ein schulischer Grundbildungsschulauftrag ist somit auf Verfassungsebene für alle minderjährigen Personen in der Schweiz gewährleistet und zwar unabhängig eines gültigen Aufenthaltsstatus. ${ }^{58}$ Diese Verfassungsbestimmungen und die gesetzliche Verankerung der Bildung als integrationsrelevante Regelstruktur ${ }^{59}$ unterstreichen die Sonderstellung des Bildungserwerbs hinsichtlich der Integration einer (minderjährigen) ausländischen Person. ${ }^{60}$

Nachfolgend werden einige Aspekte im Zusammenhang mit der öffentlichen Grundschule und der wachsenden Zahl von geflüchteten Kindern thematisiert. Der Fokus liegt auf dem Besuch der obligatorischen Grundschule von (unbegleiteten) minderjährigen Asylsuchenden, Flüchtlingen und vorläufig aufgenommenen Kindern sowie den «Sans-Papiers» Kindern.

\subsection{Schulbesuch von (unbegleiteten) minderjährigen Asylsuchenden, Flüchtlingen und vorläufig Aufgenommenen}

Der obligatorische Schulbesuch in Art. 62 Abs. 2 BV umfasst auch (unbegleitete) minderjährige Asylsuchende, Flüchtlinge und vorläufig Aufgenommene. Demnach haben (unbegleitete) minderjährige Asylsuchende einen Anspruch auf ausreichenden und unentgeltlichen Grundschulunterricht ${ }^{61}$ und allfällige sonderpädagogische Massnahmen. ${ }^{62}$ Die Umsetzung und Verwirklichung des Bildungszugangs liegt in der Zuständigkeit der Kantone. So integriert der Kanton Bern geflüchtete Kinder im Kindergartenalter direkt in den Kindergarten, einschliesslich der Unterstützung von Lektionen für Deutsch als Zweitsprache ${ }^{63}$. Ältere Kinder und Jugendliche besuchen zuerst während des Asylverfahrens und für die Dauer der Unterbringung in Kollektivunterkünften einen lokal oder regional organisierten Intensivkurs. Der Eintritt in die Regelschule erfolgt erst anschliessend. Unterricht in Deutsch als Zweitsprache wird begleitend weiterhin durchgeführt. Die Unterbringung in Wohnungen auf Gemeindeebene von Asylsuchenden und vorläufig aufgenommenen Personen erfolgt dann, wenn diese den Alltag weitgehend selbständig bestreiten können. $\mathrm{Zu}$ diesem Zeitpunkt erfolgt auch die Einschulung in die Regelklasse. Dies

\footnotetext{
56 Vgl. Art. 19 BV.

${ }^{57}$ Biaggini, G., Art. 19 Anspruch auf Grundschulunterricht, BV Kommentar, Orell Füssli Kommentar (Navigator.ch), 2.überarbeitete und erweiterte Auflage, Orell Füssli Verlag AG, Zürich, S. 255, Rn. 3, S. 256, Rn. 6, Vgl. auch Art. 28 Übereinkommen über die Rechte des Kindes (Kinderrechtskonvention, KRK), abgeschlossen in New York am 20. November 1989, In Kraft getreten für die Schweiz am 26. März 1997, SR 0.107.

58 Biaggini, (Anm. 46), S. 256, Rn. 6.

59 Art. 4 AIG, Art. 2 Abs. 3 VIntA.

${ }^{60}$ Wintsch, (Anm. 33), S. 40.

61 Vgl. Art. 19 BV.

${ }^{62}$ Vgl. Art. 62 Abs. 3 BV, Schweizerische Konferenz der kantonalen Erziehungsdirektoren (EDK), Interkantonale Vereinbarung über die Zusammenarbeit im Bereich der Sonderpädagogik, https://www.edudoc.ch/static/web/arbeiten/sonderpaed/konkordat_d.pdf (07.02.2019).

${ }^{63}$ Vgl. Erziehungsdirektion des Kantons Bern, Amt für Kindergarten, Volksschule und Beratung, Deutsch als Zweitsprache, DaZ, Ein Leitfaden zur Organisation des DaZ-Unterrichts und zur Integration von fremdsprachigen Kindern und Jugendlichen, 3. Ausgabe, Aktualisierung Dezember 2015,

https://www.erz.be.ch/erz/de/index/kindergarten_volksschule/kindergarten_volksschule/interkulturelle_bildung/deutsch _als_zweitsprache.assetref/dam/documents/ERZ/AKVB/de/01_Besondere $\%$ $\% 20$ Massnahmen/bes_massnahmen_daz_leitf aden_d.pdf (07.02.2019).
} 
geschieht gestützt auf den Stand und die Kenntnisse der Unterrichtssprache. Eine weitere Unterstützung durch Unterricht in Deutsch als Zweitsprache ist gewährleistet. ${ }^{64}$ Dieses Modell ist auch in anderen Kantonen wiederzufinden. ${ }^{65}$

Bezüglich der speziellen und vulnerablen Situation von unbegleiteten minderjährigen Asylsuchenden, verabschiedete die Konferenz der kantonalen Sozialdirektorinnen und Sozialdirektoren unverbindliche Empfehlungen für den Umgang der Kantone mit unbegleiteten minderjährigen Asylsuchenden im Jahr 2016. So soll die Einschulung so rasch als möglich erfolgen und falls notwendig die Einschulung in spezielle Klassen erfolgen. Allfällige Alphabetisierungs- oder Intensivsprachkurse sind vorrangig durchzuführen. ${ }^{66}$ Diese Empfehlungen ähneln weitestgehend der bereits bestehenden kantonalen Praxis.

Schliesslich hat die geplante Neustrukturierung und die Beschleunigung der Asylverfahren ${ }^{67}$ eine Regelung für die Zuständigkeiten für den Grundschulunterricht in den «neuen» Bundesasylzentren im Asylgesetz geschaffen. ${ }^{68}$ In den Bundeszentren sind künftig alle am Asylverfahren beteiligten Akteure untergebracht respektive vor Ort, daher die Asylsuchenden selbst, wie auch Mitarbeitende des Staatssekretariats für Migration, Rechtsberaterinnen und Rechtsberater und Betreuungs- und Sicherheitspersonal. Ziel sind schnellere und rechtsstaatliche Asylverfahren durchzuführen. ${ }^{69}$ Aktuell gibt es eine Testphase in den Bundesasylzentren seit Januar 2014 in Zürich und seit April 2019 in Boudry und Giffers. Die Umsetzung dieser Bundesasylzentren und damit beschleunige Asylverfahren ist auf März 2019 geplant. ${ }^{70}$ Während der Dauer des Asylverfahrens und der Unterbringung in den

\footnotetext{
${ }^{64}$ Vgl. Erziehungsdirektion des Kantons Bern, Amt für Kindergarten, Volksschule und Beratung, Flüchtlingskinder in der Volksschule, Informationen für Schulen und Gemeinden, 2. Fassung, November 2017, https:/www.asyl.sites.be.ch/asyl_sites/de/index/navi/index/integration/volksschule.assetref/dam/documents/ERZ/AKV B/de/09_Schulleitungen_Lehrpersonen/sl_lp_Unterlagen_asylsuchende_kinder_d.pdf (07.02.2019).

${ }^{65}$ Vgl. Kanton Zürich, Bildungsdirektion, Volksschulamt, Flüchtlingskinder in der Volksschule, Informationen für Schulen und Gemeinden, Juni 2015, https:/www.elternmitwirkung.ch/fileadmin/user_upload/Fl\%C3\%BCchtlinge/fluechtlingskinder_in_der_Volksschule_ Kanton_Z\%C3\%BCrich.pdf (07.02.2019), Kanton St. Gallen, Amt für Volksschule, Information, Kinder und Jugendliche aus dem Asylbereich in der Volksschule, 26.01.2016, https://www.schule.sg.ch/home/volksschule/schulischesumfeld/migration/_jcr_content/Par/downloadlist_1/DownloadListPar/download.ocFile/Version2_Kinder\%20und\%20Ju gendliche\%20aus\%20 $\overline{d e m} \% 20$ Asylbereich\%20in\%20der\%20Volksschule_Merkblatt.pdf (07.02.2019).

${ }^{66}$ Vgl. Konferenz der kantonalen Sozialdirektorinnen und Sozialdirektoren, Empfehlungen zu unbegleiteten minderjährigen Kindern und Jugendlichen aus dem Asylbereich, 20. Mai 2016, S. 34, http://www.sodk.ch/fileadmin/user_upload/Aktuell/Empfehlungen/2016.05.20_MNA-Empfehlungen_farbig_d.pdf (07.02.2019).

${ }^{67}$ Umsetzung ab März 2019. Vgl. Verordnung über die Durchführung von Testphasen zu den Beschleunigungsmassnahmen im Asylbereich (Testphasenverordnung, TestV) vom 4. September 2013, SR 142.318.1. ${ }^{68}$ Informationen zur Neustrukturierung und der Beschleunigung der Asylverfahren sind auf folgender Webseite zu finden: https://www.sem.admin.ch/sem/de/home/asyl/beschleunigung.html (07.02.2019).

${ }^{69} \mathrm{Vgl}$. https://www.sem.admin.ch/sem/de/home/asyl/beschleunigung/bundesasylunterkuenfte.html (07.02.2019) und die Broschüre des SEM zu den Bundesasylzentren, https://www.sem.admin.ch/dam/data/sem/asyl/broschuereasylzentren-d.pdf (07.02.2019). Vgl. auch die Kritik, Caroni, M., Scheiber N., Gutachten für die Demokratische Juristinnen und Juristen der Schweiz betreffend rechtliche Fragestellungen im Zusammenhang mit der Neustrukturierung im Asylreich und der Beschleunigung der Asylverfahren, 2015, https://www.djs-jds.ch/images/201511_FactsheetGutachtenAsyl_d.pdf (07.02.2019).

${ }^{70}$ Vgl. Verordnung über die Durchführung von Testphasen zu den Beschleunigungsmassnahmen im Asylbereich (Testphasenverordnung, TestV) vom 4. September 2013, SR 142.318.1.
} 
Bundesasylzentren, stellt der Bund in Zusammenarbeit mit dem Standortkanton den Grundschulunterricht sicher, wobei diese Aufgabe ganz oder teilweise Dritten übertragen werden kann. Grundsätzlich organisiert der Standortkanton den Schulunterricht für schulpflichtige Asylsuchende. Je nach Bedarf wird der Unterricht in den Zentren des Bundes durchgeführt. ${ }^{71}$

\subsection{Schulbesuch von «Sans-Papiers» Kindern}

Die aktuellste Studie zu Schätzungen von in der Schweiz lebenden Sans-Papiers geht von 58'000 bis 105'000 Personen aus. Die beste Schätzung dürfte 76'00 Personen sein. Es ist davon auszugehen, dass beinahe 2/3 dieser Personen ohne gültige Reisepapiere oder als Touristen und Touristinnen, mit gültigen Reisepapieren, eingereist und geblieben sind. Die restlichen Personen erhielten einen negativen Asylentscheid oder sind in der Schweiz trotz der Nichterneuerung der Aufenthalts- oder Niederlassungsbewilligung geblieben. Etwa ein Zehntel dürften minderjährige Sans-Papiers sein. Genaue Daten der Anzahl von Sans-Papiers Kindern in der öffentlichen Schule sind nicht vorhanden respektive nicht ermittelbar. ${ }^{72}$ Dennoch ist der Besuch des obligatorischen Grundschulunterrichts für Kinder ohne gültigen Aufenthalt in der Schweiz weitestgehend gewährleistet. ${ }^{73}$ Nicht zuletzt trug auch die Empfehlung der Schweizerischen Konferenz der kantonalen Erziehungsdirektoren aus dem Jahr 1991 hierzu bei: Diese betonte, dass alle fremdsprachigen Kinder in der Schweiz in die öffentliche Schule zu integrieren sind und jede Diskriminierung zu vermeiden ist. ${ }^{74}$ Am 26. Januar 2018 hat die Kommission für Soziale Sicherheit und Gesundheit des Nationalrats die Motion «Für eine kohärente Gesetzgebung zu Sans-Papiers» eingereicht. Die Motion verlangte unter anderem die Meldepflicht von Schulbehörden bei Sans-Papiers Kindern. Die Motion wurde am 18. Mai 2018 zurückgezogen. ${ }^{75}$ Im Zuge der Teilrevision des Ausländergesetzes, ab 1. Januar 2019 neu betitelt als Ausländerinnen-, Ausländer- und Integrationsgesetz (AIG) in $\mathrm{Kraft}^{76}$, wurde die Verordnung über Zulassung, Aufenthalt und Erwerbstätigkeit ${ }^{77}$ angepasst. Seit dem 1. Januar 2019 haben die Schulbehörden eine Meldepflicht gegenüber den kantonalen Schulbehörden bezüglich definitiven Schulausschlüssen von ausländischen Schülerinnen und Schülern. ${ }^{78}$ Aber Art. 82e Abs. 2 VZAE schliesst die Meldepflicht aus, wenn die betroffene Schülerin respektive der betroffene Schüler keinen

\footnotetext{
${ }^{71}$ Vgl. Art. 80 Abs. 1 AsylG, auch Konferenz der kantonalen Sozialdirektorinnen und Sozialdirektoren, Konferenz der Kantonalen Justiz- und Polizeidirektorinnen und-direktoren, Staatssekretariat für Migration, Faktenblatt zur Neustrukturierung Asyl, 10 Grundschulunterricht für schulpflichtige Asylsuchende in den Bundesasylzentren, Stand Juli 2017, https://www.sem.admin.ch/dam/data/sem/asyl/beschleunigung/infoveranstaltungen/themen/10grundschulunterricht-d.pdf (07.02.2019).

${ }^{72}$ B, S, S. Volkswirtschaftliche Beratung in Kooperation mit SFM und Universität Genf, Sans-Papiers in der Schweiz 2015, Basel, 12. Dezember 2015, https://www.sem.admin.ch/dam/data/sem/internationales/illegalemigration/sans_papiers/ber-sanspapiers-2015-d.pdf(07.02.2019), S. 1, 8, 71, 72.

73 Art. 28 KRK, Biaggini, (Anm. 46), S. 256, Rn. 6, Petry, P., La situation juridique des migrants sans statut légal, Entre droit international des droits de l'homme et droit suisse des migrations, Collection genevoise, Schulthess Editions romandes, Zürich, 2013, S. 274.

${ }^{74}$ Schweizerische Konferenz der kantonalen Erziehungsdirektoren, Empfehlungen zur Schulung der fremdsprachigen Kinder vom 24. Oktober 1991, https://edudoc.ch/record/24317/files/EDK-Empfehlungen_d.pdf (07.02.2019).

${ }^{75}$ Vgl. Kommission für Soziale Sicherheit und Gesundheit Nationalrat, 18.3005 Motion, Für eine kohärente Gesetzgebung zu Sans-Papiers, 26.01.2018, https://www.parlament.ch/de/ratsbetrieb/suche-curiavista/geschaeft?AffairId=20183005 (07.02.2019).

76 AS 20176521.

${ }^{77}$ Verordnung über Zulassung, Aufenthalt und Erwerbstätigkeit vom 24. Oktober 2007 (VZAE), SR 142.201.

${ }^{78}$ Art. 82e VZAE.
} 
rechtmässigen Aufenthalt in der Schweiz hat. ${ }^{79}$ Aktuell verlangt ein Postulat eine Gesamtprüfung der Problematik der Sans-Papiers. ${ }^{80}$

\section{Nachobligatorische Schulbildung: Zugang zu den Hochschulen}

Geflüchtete Personen haben aus rechtlicher Sicht grundsätzlich die Möglichkeit eine nachobligatorische Schulbildung wie das Studium an einer Universität oder Fachhochschule. Da es keine zentrale Zulassungsstelle für ein Studium an einer schweizerischen Hochschule gibt, ist jede Hochschule selber dafür zuständig. ${ }^{81}$ Ein Recht für die Zulassung zu einem Studium gibt es somit nicht. Geflüchtete Personen, die studieren möchten, müssen sich daher direkt an die Hochschule wenden. Der Einbezug des Aufenthaltsstatus einer geflüchteten Person kann dabei bei der Zulassung zum Studium eine Rolle spielen ${ }^{82}$ mit Ausnahme von anerkannten Flüchtlingen respektive vorläufig Aufgenommenen mit Flüchtlingsstatus. ${ }^{83}$

Jedoch gestaltet sich die tatsächliche Zulassung zu einem Studium an einer der schweizerischen Hochschulen als schwierig. Zahlreiche Hürden wie fehlende oder ungenügende Sprachkenntnisse, die Nichtanerkennung der bisherigen Schulbildung ${ }^{84}$, fehlende finanzielle Mittel und schliesslich die Unübersichtlichkeit des Schweizer Bildungssystems aufgrund der föderalen Zuständigkeiten und des Hochschulsystems erschweren geflüchteten Personen sich zu informieren. Der Verband der Schweizerischen Studierendenschaft lancierte aus diesem Grund eine Informationswebseite, welche Informationen zu den Hochschulen in der Schweiz für geflüchtete Personen enthält. ${ }^{85}$ Zusätzlich gibt es an einzelnen Hochschulen zivilgesellschaftliche Initiativen, welche geflüchtete Personen beim Prozess für die Zulassung zum Studium begleiten. ${ }^{86}$ Auch haben teilweise die Universitäten spezifische Informationen für geflüchtete Personen bereitgestellt. ${ }^{87}$ Dennoch bleibt der tatsächliche Zugang schwierig, unter anderem auch weil die Finanzierung nicht einfach respektive sich als schwierig gestaltet. ${ }^{88}$ Denn das Stipendienwesen des Studiums ist kantonal geregelt und die betroffene Person ist, je nach Aufenthaltsstatus, nicht antragsberechtigt. Beispielsweise verlangen die Kantone Bern und Zürich für die Beantragung eines Stipendiums die Anerkennung als Flüchtling und ein fünfjähriger Wohnsitz im Kanton. ${ }^{89}$ Hingegen ermöglicht der Kanton Waadt ein Stipendium auch

\footnotetext{
${ }^{79}$ Vgl. Art. 82e Abs. 2 VZAE.

${ }^{80}$ Vgl. Staatspolitische Kommission Nationalrat, 18.3381 Postulat, Gesamte Prüfung der Problematik der Sans-Papiers vom 12.04.2018, https://www.parlament.ch/de/ratsbetrieb/suche-curia-vista/geschaeft?AffairId=20183381 (07.02.2019).

${ }^{81}$ Vgl. www.swissuniversitites.ch (07.02.2019).

${ }^{82}$ Vgl. www.perspektiven-studium.ch (07.02.2019).

${ }^{83}$ Vgl. Art. 22 Abs. 2 Abkommen über die Rechtstellung der Flüchtlinge, abgeschlossen in Genf am 28. Juli 1951, In Kraft getreten für die Schweiz am 21. April 1955, SR 0.142.30.

${ }^{84}$ Vgl. Sontag, K., Highly Skilled Asylum Seekers: Case studies of refugee students at a Swiss university, Migration Letters, October 2018, S. 538.

${ }^{85}$ Vgl. (Anm. 69), Die Webseite ist auf Deutsch, Französisch und Englisch.

${ }^{86}$ Vgl. Universität Basel, www.offener-hoersaal.ch, Universität Bern, https://www.sub.unibe.ch/de/Dienstleistungen/Offener-Hoersaal (07.02.2019).

${ }^{87}$ Vgl. Universität Zürich, https:/www.int.uzh.ch/de/in/refugees.html, Eidgenössische Technische Hochschule Zürich, https:/www.ethz.ch/en/studies/international-immigration-housing/refugees.html (07.02.2019).

${ }^{88}$ Vgl. Sontag, K., (Anm. 71), S. 540.

${ }^{89}$ Vgl. Bildungsdirektion Kanton Zürich, Merkblatt Ausländer/innen, https://ajb.zh.ch/internet/bildungsdirektion/ajb/de/stipendien/voraussetzungen/wohnsitz/_jcr_content/contentPar/downlo
} 
für vorläufig Aufgenommene Personen ohne Flüchtlingsstatus, sofern die Eltern finanziell unabhängig sind. ${ }^{90}$ Asylsuchende sind von einem kantonalen Stipendium ausgeschlossen. ${ }^{91}$ Hier gibt es jedoch, wenn auch nur wenige, private Stiftungen, die unter Umständen einen finanziellen Beitrag für ein Studium gewährleisten. ${ }^{92}$ Schliesslich ist eine alternative Möglichkeit zur Zulassung zum Studium, die Registrierung als Gasthörerin respektive Gasthörer an einer der Hochschule in der Schweiz. ${ }^{93}$

\section{Berufliche Ausbildung}

Bestimmungen über die berufliche Ausbildung erlässt der Bund. ${ }^{94}$ Eine Besonderheit der Schweiz ist das duale Ausbildungssystem im Berufsbildungsprozess. Schulische Theorie und praktische Berufserfahrung werden verknüpft um eine berufliche Grundausbildung zu erwerben. ${ }^{95}$ Um eine Berufsausbildung ${ }^{96}$ absolvieren zu können, muss ein Lehrvertrag abgeschlossen werden. Dies bedingt den Abschluss eines Arbeitsvertrags, respektive den Besitz einer Arbeitsbewilligung ${ }^{97}$, was wiederum mit dem Besitz eines gültigen Aufenthaltsstatus ${ }^{98}$ verknüpft ist. Somit haben Asylsuchende grundsätzlich keine Möglichkeit eine berufliche Ausbildung zu absolvieren, da bei einem negativen Entscheid die Ausbildung abgebrochen werden muss. Dennoch sind Ausnahmen möglich. Aufgrund des Vorrangs auf dem Arbeitsmarkt von Schweizerinnen und Schweizer, sowie Personen mit einem Aufenthaltsstatus C, B oder $\mathrm{F}^{99}$, muss zuerst bewiesen werden, dass keine Person gefunden wurde, die unter diese inländische Arbeitnehmerschaft fällt. Zusätzlich muss eine Bestätigung des regionalen Arbeitsvermittlungsamts vorliegen, das die offene Lehrstelle gemeldet wurde, und das Inserate auf den einschlägigen Webseiten oder bei den Berufsverbänden veröffentlicht wurden. Diese Nachweise müssen erbracht werden, damit der Beweis erbracht wird, dass keine Person mit Vorrang gefunden wurde. Anschliessend kann ein Gesuch, mit dem entsprechenden Lehrvertrag und eventuell weiteren geforderten Unterlagen bei den zuständigen kantonalen Behörden eingereicht werden. Diese entscheiden schliesslich über die Erteilung einer Bewilligung zur Erwerbstätigkeit. Jedoch gilt auch in einem solchen Sachverhalt, dass bei einem negativen Asylentscheid mit einem rechtskräftigen Wegweisungsentscheid die Ausbildung abgebrochen werden muss, da die Bewilligung der Erwerbstätigkeit erlischt. ${ }^{100}$ Jedoch kann das Eidgenössische Justiz- und Polizeidepartement mit dem

adlist_1/downloaditems/merkblatt_ausl_nderi.spooler.download.1339142506406.pdf/Merkblatt_AuslaenderInnen.pdf (07.02.2019), Erziehungsdirektion des Kantons Bern, Stipendien und Darlehen, Informationen über

Ausbildungsbeiträge, Ausgabe November 2017,

https:/www.erz.be.ch/erz/de/index/hochschule/hochschule/stipendien_und_darlehen/allgemeine_informationen.assetref /dam/documents/ERZ/AZD/de/Stipendien\%20und\%20Darlehen\%202016.pdf (07.02.2019).

${ }^{90} \mathrm{Vgl}$. Art. 8 Abs. 1 let.g Loi du 1 juillet 2014 sur l'aide aux études et à la formation professionnelle (LAEF), 416.11.

${ }^{91}$ Vgl. http://www.migraweb.ch/de/themen/asylrecht/aufenthalt/bildung/ (07.02.2019).

${ }^{2} \mathrm{Vgl}$. das Stiftungsverzeichnis der Schweiz, https:/www.edi.admin.ch/edi/de/home/fachstellen/eidgenoessische-

stiftungsaufsicht/stiftungsverzeichnis.html (07.02.2019).

93 Vgl. (Anm. 69).

94 Art. 63 Abs. 1 BV, Bundesgesetz über die Berufsbildung vom 13. Dezember 2002 (Berufsbildungsgesetz, BBG), SR 412.10 .

${ }^{95}$ Siehe Berufsbildungsgesetz.

${ }^{96}$ In der Schweiz spricht man von einer Lehre, Lehrstelle, Lehrbetrieb etc.

${ }^{97}$ Vgl. Art. 43 AsylG, Art. 61 AsylG, Art. 11 AIG.

98 Vgl. Art. 60 AsylG, Art. 85 Abs. 6 AIG.

${ }^{99} \mathrm{Vgl}$. Art. 21 Abs. 2 AIG.

${ }^{100}$ Vgl. Art. 43 Abs. 2 AsylG.. 
Departement für Wirtschaft, Bildung und Forschung die Kantone ermächtigen, für bestimmte Kategorien von Personen die Arbeitsbewilligung über den Ablauf der Ausreisefrist hinaus zu verlängern. Dies muss durch besondere Umstände gerechtfertigt sein. ${ }^{101}$ Die Erwerbstätigkeit von Asylsuchenden ist jedoch nicht das Ziel, solange das Asylverfahren noch hängig ist. Der Vollzug der Wegweisung nach einem negativen Asylentscheid darf somit durch die Erteilung einer Erwerbstätigkeit nicht behindert werden. ${ }^{102}$

Bereits diese Ausführungen lassen darauf schliessen, dass geflüchtete Personen nur unter erschwerten Bedingungen Zugang zu einer Berufsausbildung haben. Ergänzt wird diese Hürde durch eine teilweise fehlende schulische Grundausbildung und ungenügende Sprachkenntnisse. Nachfolgend wird auf einige Aspekte, wie die spezielle Regelung für die Berufsausbildung von Sans-Papiers und das Pilotprogramm „Integrationsvorlehre“ hingewiesen. Abschliessend wird die Integrationsagenda Schweiz thematisiert, die beide eine nachhaltige Arbeitsmarktintegration zum Ziel haben.

\subsection{Berufsausbildung für Sans-Papiers-Jugendliche}

Seit dem 1. Februar 2013 besteht für Sans-Papiers-Jugendliche die Möglichkeit für die Dauer der Berufslehre ein befristetes Aufenthaltsrecht zu erhalten. ${ }^{103}$ Eine Aufenthaltsbewilligung für eine berufliche Grundausbildung kann für Sans-Papiers-Jugendliche unter folgenden Voraussetzungen erteilt werden:

«Die Gesuchstellerin oder der Gesuchsteller hat die obligatorische Schule während mindestens fünf Jahren ununterbrochen in der Schweiz besucht und reicht danach innerhalb von zwölf Monaten ein Gesuch ein; die Teilnahme an Brückenangeboten ohne Erwerbstätigkeit wird an die obligatorische Schulzeit angerechnet; das Gesuch des Arbeitgebers nach Artikel 18 Buchstabe b AIG ${ }^{104}$ liegt vor; die Lohn- und Arbeitsbedingungen nach Artikel $22 \mathrm{AIG}^{105}$ werden eingehalten; die Gesuchstellerin oder der Gesuchsteller ist gut integriert; sie oder er respektiert die Rechtsordnung; sie oder er legt ihre Identität offen ${ }^{106}$.

Besonders die Offenlegung der Identität der Gesuchstellerin respektive des Gesuchstellers birgt Schwierigkeiten. Denn dadurch kann auch die Bekanntgabe allfälliger Familienmitglieder folgen, denen je nach Umständen die Ausweisung droht. Die Eltern sowie die Geschwister haben jedoch die Möglichkeit eine Aufenthaltsbewilligung $\mathrm{zu}$ erhalten, sofern die Voraussetzungen des schwerwiegenden persönlichen Härtefalls gegeben sind. ${ }^{107}$ Da kein Anspruch auf Erteilung einer

\footnotetext{
${ }^{101}$ Vgl. Art. 43 Abs. 3 AsylG.

${ }^{102} \mathrm{Vgl}$. die Ausführungen des Staatssekretariates für Wirtschaft (SECO), https://www.seco.admin.ch/seco/de/home/Arbeit/Personenfreizugigkeit_Arbeitsbeziehungen/schwarzarbeit/Arbeit_korr ekt_melden/Pflichten_Arbeitgebenden/Auslaenderrecht/Personen_Asylbereich.html\#-119204595 (07.02.2019).

${ }^{103}$ Die Regelung geht auf Luc Barthassat, 08.3616 Motion, Jugendlichen ohne gesetzlichen Status eine Berufslehre ermöglichen, 2.10.200, zurück.

104 Art. 18 let. b AIG: Ausländerinnen und Ausländer können zur Ausübung einer unselbständigen Erwerbstätigkeit zugelassen werden, wenn: b. das Gesuch eines Arbeitgebers vorliegt.

${ }^{105}$ Art. 22 AIG: Ausländerinnen und Ausländer können zur Ausübung einer Erwerbstätigkeit nur zugelassen werden, wenn die orts-, berufs- und branchenüblichen Lohn- und Arbeitsbedingungen eingehalten werden.

106 Art. 30a Abs. 1 let. a-f Verordnung über Zulassung, Aufenthalt und Erwerbstätigkeit vom 24. Oktober 2007 (VZAE), SR 142.201.

107 Siehe die Voraussetzungen in Art. 31 VZAE.
} 
Aufenthaltsbewilligung für die / den Sans-Papiers im Jugendalter besteht ${ }^{108}$, ist die Eingabe eines Gesuches entsprechend sorgfältig zu prüfen. Seit Inkrafttreten der Regelung, 2013, sind bis anhin 35 Gesuche beim Staatssekretariat für Migration eingegangen. ${ }^{109}$

\subsection{Förderung der nachhaltigen beruflichen Eingliederung}

Aufgrund des neuen Ausländerinnen- und Ausländer- und Integrationsgesetz (AIG) und die darin enthaltene Umsetzung von Art. 121a BV, sowie andererseits durch die Neustrukturierung und Beschleunigung der Asylverfahren, hat der Bund, gemeinsam mit den Kantonen und anderen Sozialpartnern, zwei Projekte angestossen, welche die nachhaltige berufliche Eingliederung von geflüchteten Personen zum Ziel haben. Es handelt sich hierbei um das Pilotprogramm «Integrationsvorlehre» und die «Integrationsagenda Schweiz».

\subsubsection{Pilotprogramm «Integrationsvorlehre»}

Im Dezember 2015 kündigte der Bundesrat Begleitmassnahmen ${ }^{110}$ zur Umsetzung der angenommenen Volksinitiative «gegen Masseneinwanderung» ${ }^{111}$ an. Nebst dem Teilprojekt der sprachlichen Frühförderung von Asylsuchenden mit Bleibeperspektive, bildet das Absolvieren einer einjährigen Integrationsvorlehre für 800 bis 1000 Personen pro Jahr einen zweiten Schwerpunkt. Das Ziel ist, dass die teilnehmenden anerkannten Flüchtlinge und die vorläufig Aufgenommenen die notwendigen grundlegenden Kompetenzen hinsichtlich einer beruflichen Grundausbildung erwerben. Der Erwerb folgender Kompetenzen ist vorgesehen: «sprachliche Kompetenzen in der jeweiligen Landessprache, schulische Grundkompetenzen, Normen und Werte (kulturelle Kompetenzen); wichtige überfachliche Kompetenzen (z.B. Sozial- und Selbstkompetenzen mit Bezug zum Berufsfeld, Lerntechnik), berufsfeldbezogene Grundfertigkeiten und Grundlagenwissen, Arbeitserfahrungen in einem Betrieb im angestrebten Berufsfeld in der Schweiz» ${ }^{112}$.

Der Abschluss der Integrationsvorlehre soll möglichst viele Teilnehmende praktisch, wie schulisch, für eine berufliche Grundausbildung vorbereiten. ${ }^{113}$ Seit dem Sommer 2018 bieten 18 Kantone eine einjährige Integrationsvorlehre an und haben entsprechende Verträge mit dem Staatssekretariat für Migration abgeschlossen. ${ }^{114}$ Die Idee der Integrationsvorlehre ist in der Schweiz nicht neu. Bereits 2006 wurde ein ähnliches Projekt unter dem Namen «Flüchtlingsanlehre» getestet, wobei die

\footnotetext{
108 Petry, R., Die rechtliche Bewältigung irregulärer Migration: Die Situation der «Sans-Papiers», in: Achermann, Amarelle, Caroni, Epiney, Kälin, Uebersax (Hrsg.), Jahrbuch für Migrationsrecht 2014/2015, Stämpfli Verlag AG, Bern, 2015, S. 15.

${ }^{109}$ Die Angabe bezieht auf die Webseite der Eidgenössischen Migrationskommission, https://www.ekm.admin.ch/ekm/de/home/zuwanderung---aufenthalt/sanspapiers/berufslehre.html (07.02.2019).

${ }^{110}$ Bundesrat, (Anm. 16).

111 Art. 121a BV.

112 Staatsekretariat für Migration, Eckpunkte Pilotprogramm «Integrationsvorlehre», 6.09.2016, https:/www.sem.admin.ch/dam/data/sem/integration/ausschreibungen/2018-integrvorlehre/eckpunkte-invol-d.pdf (07.02.2019).

113 Staatsekretariat für Migration, Eckpunkte Pilotprogramm «Integrationsvorlehre», 6.09.2016.

${ }^{114}$ Vgl. https:/www.sem.admin.ch/sem/de/home/aktuell/news/2018/2018-05-17.html (07.02.2019), Kurt, S., A Modest Start: Integration Policies in the Field of Asylum, in: NCCR on the move (Hrsg.), Highlights \#2, S. $17 \mathrm{f}$.
} 
Evaluation nicht nur positiv ausfiel. Insbesondere die Suche nach beruflichen Anschlusslösungen stellten sich als grosse Herausforderung dar. ${ }^{115}$

\subsubsection{Integrationsagenda Schweiz}

Die Integrationsagenda Schweiz entstand in Zusammenarbeit des Bundes und der Kantone aufgrund der Neustrukturierung und der Beschleunigung der Asylverfahren, die im März 2019 umgesetzt werden. ${ }^{116}$ Diese Integrationsagenda wird im Rahmen der bestehenden kantonalen Integrationsprogramme umgesetzt. ${ }^{117}$ Sie umfasst fünf Wirkungsziele für Flüchtlinge und vorläufig Aufgenommene, wobei der Hauptschwerpunkt auf die sprachliche wie berufliche Bildung gelegt ist. So sollen Flüchtlinge und vorläufig Aufgenommene drei Jahre nach der Einreise über mindestens das sprachliche Niveau A1 des Gemeinsamen Europäischen Referenzrahmen (GER) für Sprachen verfügen. Weiter sollen zwei Drittel aller Flüchtlinge und vorläufig Aufgenommenen zwischen 1625 Jahre fünf Jahre nach der Einreise sich in einer postobligatorischen Ausbildung (Berufsausbildung respektive Hochschulen) befinden. Schliesslich soll die Hälfte der erwachsenen Flüchtlinge und vorläufig Aufgenommene sieben Jahre nach der Einreise nachhaltig in den Arbeitsmarkt integriert sein. ${ }^{118}$ Ein einheitlicher Integrationsprozess für Flüchtlinge und vorläufig Aufgenommene soll die Erreichung dieser Ziele unterstützen. Als erstes werden Erstinformationsgespräche durchgeführt, sowie eine Potenzialabklärung vorgenommen. In den Erstinformationsgesprächen werden anerkannte Flüchtlinge sowie vorläufig Aufgenommene persönlich über die Gepflogenheiten, die Regelung und die Unterstützungsangebote informiert. Die Potenzialabklärung erfolgt systematisch und jede Person wird individuell hinsichtlich der Wirtschaft und der Gesellschaft gefördert, um den grössten Nutzen für alle Akteure zu erreichen. Parallel erfolgt frühzeitig das Erlernen einer Landessprache durch gezielte Sprachkurse. Konkret sollen bildungsfähige Jugendliche auf eine Ausbildung vorbereitet werden und dann diese auch in Angriff nehmen können. Arbeitsfähige Erwachsene sollen für den Arbeitsmarkt fit gemacht werden und für die übrigen Personen ist der Schwerpunkt auf die soziale Integration gelegt. Durchgehend erfolgt eine enge Fallführung. ${ }^{119}$ Für die Umsetzung wird neu die Integrationspauschale von bisher CHF 6'000 auf CHF $18^{\prime} 000$ erhöht. ${ }^{120}$ Die Umsetzung der Integrationsagenda ist auf September 2019 zu erwarten. ${ }^{121}$

\footnotetext{
${ }^{115}$ Vgl. Staatssekretariat für Migration, KEK/CDC Consultants, Pilote Berufliche Integration für Flüchtlinge des BFM, Gesamtschlussbericht über die 3 Pilote «Logistik», «Overall» und «Gastro», September 2008, https://www.sem.admin.ch/dam/data/sem/integration/berichte/va-flue/ber-pilot-d.pdf (07.02.2019).

${ }^{116}$ Vgl. Eidgenössisches Justiz- und Polizeidepartement, Eidgenössisches Departement für Wirtschaft, Bildung und Forschung, Konferenz der Kantonsregierungen, Schweizerische Konferenz der kantonalen Erziehungsdirektoren, Konferenz der kantonalen Sozialdirektorinnen und Sozialdirektoren, Faktenblatt, Die Integrationsagenda kurz erklärt, 25.04.2018, https://www.sem.admin.ch/dam/data/sem/integration/agenda/faktenblatt-integrationsagenda-d.pdf (07.02.2019).

${ }^{117}$ Vgl. Staatssekretariat für Migration, Rundschreiben, Eckwerte zur Planung der Umsetzung der Integrationsagenda Schweiz im Rahmen der KIP 2018-2012, 5. Juli 2018, https://www.sem.admin.ch/dam/data/sem/integration/agenda/20180705-rs-planung-umsetzung-ias-d.pdf (07.02.2019). 118 Vgl. Eidgenössisches Departement für Wirtschaft, Bildung und Forschung, (Anm. 41), S. 11,12.

${ }^{119}$ Vgl. http://www.kip-pic.ch/de/kip/integrationsagenda/ (07.02.2019).

${ }^{120}$ Vgl. Integrationsagenda Schweiz, Bericht der Koordinationsgruppe vom 1. März 2018, S. 13.

${ }^{121}$ Vgl. Staatssekretariat für Migration, Rundschreiben, Eckwerte zur Planung der Umsetzung der Integrationsagenda Schweiz im Rahmen der KIP 2018-2012, 5. Juli 2018.
} 


\section{Schlussbemerkungen}

Ende Dezember 2017 hielten sich 121'402 Personen, resultierend aus dem Asylbereich, in der Schweiz auf. Davon waren 51'512 anerkannte Flüchtlinge und 41'544 vorläufig aufgenommene Personen. ${ }^{122}$ 24'231 Personen sind statistisch als Asylsuchende ausgewiesen. Die Alterspyramide bei den Asylsuchenden zeigt, dass die Mehrheit zwischen 20 bis 24 Jahre alt ist, wobei aber auch bei den 0 bis 4-jährigen rund 1'220 Personen verzeichnet sind. Bei den vorläufig aufgenommenen Personen zeigt sich eine ähnliche Altersstruktur, wobei hier die höchste Anzahl der Personen zwischen 15-19jährig ist. ${ }^{123}$ Diese rudimentären Zahlen bilden lediglich ein Indiz dafür, wie wichtig der rechtliche, aber schliesslich der tatsächliche Zugang zu Bildung für Personen aus dem Asylbereich ist. Ebenso verweist die Integrationsagenda Schweiz auf die stärkere Zuwanderung von jungen Menschen in die Schweiz und dem entsprechenden Bedarf nachhaltige Bildungsangebote zu schaffen. ${ }^{124}$

Allerdings ist auf die ungleiche Ausgestaltung hinsichtlich des Bildungszugangs von geflüchteten Personen hinzuweisen. Diese beruhen einerseits auf der föderalen Ausgestaltung des Bildungsbereiches und sind andererseits auf das zugrundeliegende Verständnis der Integrationspolitik in der Schweiz zurückzuführen. Während (unbegleitete) minderjährige Asylsuchende, wie auch Flüchtlinge und vorläufig Aufgenommene im schulpflichtigen Alter die Grundschule besuchen können, gibt es nur wenige Angebote für volljährige Asylsuchende. In den Empfangs- und Verfahrenszentren besteht die Möglichkeit an Beschäftigungsprogrammen teilzunehmen und falls eine Bleibeperspektive gegeben ist, die Teilnahme am Programm der frühen Sprachförderung. Für die Absolvierung einer Berufslehre sind die Hürden sehr hoch. Schliesslich erweist sich auch der Weg zur Zulassung an einer der Schweizer Hochschulen als schwierig. Aufgrund dieser Angebotslücken bestehen daher einige zivilgesellschaftliche Initiativen, welche unterschiedliche Bildungsaktivitäten anbieten.

Aus rechtlicher Sicht ändert sich die Situation für geflüchtete Personen erst ab dem Zeitpunkt, wenn ein «gefestigter» Aufenthaltsstatus, wie die Anerkennung als Flüchtling oder eine vorläufige Aufnahme erfolgt. Ab diesem Zeitpunkt beginnen aus rechtlicher Sicht die spezifischen Integrationsförderungsmassnahmen. Die Möglichkeit der Teilnahme am Programm der einjährigen Integrationsvorlehre respektive die Absolvierung einer Berufslehre stehen nun offen und unter Umständen ist auch der Zugang zu einer Hochschule eher möglich, da durch den «gefestigten» Aufenthaltsstatus bessere Chancen auf eine Finanzierungsmöglichkeit bestehen. Die kürzlich initiierten Programme durch den Bund, in Zusammenarbeit mit den Kantonen und anderen Akteurinnen und Akteuren, sind denn auch ein deutliches Zeichen, dass Handlungsbedarf im Bereich Zugang zur Bildung von geflüchteten Personen besteht. Inwiefern diese Initiativen die Zielsetzungen erfüllen, bleibt abzuwarten.

\section{Autorin}

\footnotetext{
122 Hinzukommen noch Personen, deren Wegweisungsvollzug ausgesetzt wurde oder auch Spezialfälle. Für eine detaillierte Auflistung, siehe SEM, Kommentierte Asylstatistik 2017, 22. Januar 2018, https:/www.sem.admin.ch/dam/data/sem/publiservice/statistik/asylstatistik/2017/stat-jahr-2017-kommentar-d.pdf (07.02.2019).

${ }^{123}$ Vgl. SEM, Kommentierte Asylstatistik 2017, 22. Januar 2018.

${ }^{124}$ Vgl. Integrationsagenda Schweiz, Bericht der Koordinationsgruppe vom 1. März 2018.
} 
Stefanie Kurt, Assistenzprofessorin FH (Tenure Track), Dr.iur., Hochschule für Soziale Arbeit, HES-SO Valais-Wallis, Route de la Plaine 2, 3960 Siders, 00412760691 00, stefanie.kurt@hevs.ch 